\title{
Fixed point theory for homogeneous spaces, II
}

\author{
by \\ Peter Wong (Lewiston, ME)
}

\begin{abstract}
Let $G$ be a compact connected Lie group, $K$ a closed subgroup and $M=$ $G / K$ the homogeneous space of right cosets. Suppose that $M$ is orientable. We show that for any selfmap $f: M \rightarrow M, L(f)=0 \Rightarrow N(f)=0$ and $L(f) \neq 0 \Rightarrow N(f)=R(f)$ where $L(f), N(f)$, and $R(f)$ denote the Lefschetz, Nielsen, and Reidemeister numbers of $f$, respectively. In particular, this implies that the Lefschetz number is a complete invariant, i.e., $L(f)=0$ iff $f$ is deformable to be fixed point free. This was previously known under the hypothesis that $p_{*}: H_{n}(G) \rightarrow H_{n}(M)$ is nontrivial where $n=\operatorname{dim} M$. A simple formula using equivariant degree is given for the Reidemeister trace of a selfmap $f: M \rightarrow M$.
\end{abstract}

1. Introduction. Let $M$ be a closed connected triangulated orientable manifold of dimension $\geq 3$. For any selfmap $f: M \rightarrow M$, there is only one obstruction (primary), denoted by $o(f)$, to deforming $f$ to be fixed point free. If $L(f)=0 \Rightarrow o(f)=0$, where $L(f)$ denotes the Lefschetz number of $f$, then the converse of the Lefschetz fixed point theorem holds true. In other words, the Lefschetz number would be a complete invariant. The vanishing of $o(f)$ is equivalent to the vanishing of the Nielsen number $N(f)$ which gives a sharp lower bound for the number of fixed points of maps in the homotopy class of $f$. The relationship between $o(f)$ and $N(f)$ was given explicitly by E. Fadell and S. Husseini in [4]. Since $N(f)$ is defined to be the number of fixed point classes of nonzero fixed point index and the Lefschetz number is the sum of the fixed point indices of the fixed point classes, $L(f)=0$ implies $N(f)=0$ when the fixed point classes have index of the same sign. In fact, $L(f) \neq 0 \Rightarrow N(f)=R(f)$ where $R(f)$ denotes the Reidemeister number defined at the fundamental group level. It is therefore desirable to

2000 Mathematics Subject Classification: Primary 55M20; Secondary 57S99.

Key words and phrases: Lefschetz number, Nielsen number, Reidemeister trace, fixed points, root theory, homogeneous spaces, equivariant maps, equivariant obstruction, Bredon-Illman cohomology. 
find spaces $M$ for which

$$
\begin{aligned}
& L(f)=0 \Rightarrow N(f)=0, \\
& L(f) \neq 0 \Rightarrow N(f)=R(f),
\end{aligned}
$$

for all selfmaps $f: M \rightarrow M$. We call such spaces Jiang type spaces. In [16], we employed the Nielsen root theory of R. Brooks [1] to further explore the connection, made explicit by E. Fadell in [3], between the fixed point problem for orientable homogeneous spaces and the associated Borsuk-Ulam type problem. In particular, we showed that an orientable homogeneous space $M=G / K$, where $G$ is a compact connected Lie group and $K$ a closed subgroup, is a Jiang type space provided the homomorphism $p_{*}$ : $H_{n}(G) \rightarrow H_{n}(M)$ induced by the projection map on the $n=\operatorname{dim} M$-th integral homology is nonzero. When $K$ is connected, it is well known that $M=G / K$ is a Jiang space. The main objective of this paper is to prove the same result (Theorem 2.2) without the hypothesis on $p_{*}$. Thus, our result implies that for orientable coset spaces of compact connected Lie groups, the Lefschetz number is a complete invariant. Analogously, it is known that if $M_{1}$ and $M_{2}$ are closed connected orientable triangulated manifolds of the same dimension then the topological degree is a complete invariant, i.e., for any $f: M_{1} \rightarrow M_{2}$ and $e \in M_{2}, \operatorname{deg} f \neq 0$ iff $g^{-1}(e) \neq \emptyset$ for any $g \sim f$.

First consider the following example in which $p_{*}$ is trivial.

ExAmple 1.1. Let $p: S^{3} \rightarrow S^{2}$ be the Hopf map. Consider $S^{2}=S^{3} / S^{1}$ as a coset space and let $[e] \in S^{2}$ be the coset of the identity element $e \in S^{3}$. Then $p$ has exactly one root class, namely $S_{[e]}^{1}=p^{-1}([e])$, whose root index $\omega\left(p, S_{[e]}^{1}\right)$ is the zero homomorphism but $S_{[e]}^{1}$ is essential because $p$ is not null-homotopic.

Consider $\varphi(g)=[e]$, the constant map at [e]. The subgroup $S^{1}$ acts on $S^{2}$ via left translation and it acts on $S^{3}$ via $k * g=g k^{-1}$ for $k \in S^{1}$ and $g \in S^{3}$. Now $\varphi$ becomes an $S^{1}$-equivariant map and it has one root class $\alpha=S^{3}$ which is inessential as an ordinary root class but it cannot be removed by any $S^{1}$-homotopy.

In this example, the secondary obstruction to deforming $p$ to be root free is nontrivial while the primary one vanishes. Thus $p$ cannot be homotopic to any map $q$ with $q^{-1}([e])=\emptyset$. For the map $\varphi$, the ordinary root index $\omega\left(\varphi, S^{3}\right)$ (see [1] or [16]), which can be viewed as the primary obstruction to deforming $\varphi$ to be root free, is zero. Furthermore, the secondary obstruction, which lies in $H^{3}\left(S^{3} ; \pi_{3}\left(S^{2}\right)\right)$, is also trivial. Hence, $\varphi$ is homotopic to a map $\psi$ such that $\psi^{-1}([e])=\emptyset$. On the other hand, when we consider the deformation problem for $\varphi$ in the $S^{1}$-equivariant homotopy class, there is only one (primary) equivariant obstruction and it is nontrivial. This simple example suggests that the hypothesis $p_{*} \neq 0$ need not be necessary and 
equivariant obstruction may be used to handle the situation when $p_{*}$ is zero. Since the assumption $p_{*} \neq 0$ was used in [16] to show that the ordinary root index was nonzero, which then implied the equivariant root index to be nonzero, we must by-pass the use of the ordinary root index and instead give a direct proof of the nonvanishing of the equivariant root index.

Throughout we assume that $G$ is a compact connected Lie group and $K$ is a closed but not necessarily connected subgroup. In addition, we assume that the homogeneous space $M=G / K$ of right cosets is orientable. The subgroup $K$ acts freely on $G$ via $k * g=g k^{-1}$ for $k \in K$ and $g \in G$. It also acts on $M$ via $k \circ(g K)=k g K$. Given any selfmap $f: M \rightarrow M$, there is an associated $K$-equivariant map $\varphi: G \rightarrow M$ given by $\varphi(g)=g^{-1} f(g K)$. In fact, this association is a 1-1 correspondence between the selfmaps of $M$ and the set of $K$-maps from $G$ to $M$ (see [3]). Note that $f(g K)=g K$ iff $\varphi(g)=e K$ where $e \in G$ denotes the identity element in $G$. Thus, the fixed point problem of a map $f: M \rightarrow M$ is equivalent to the $K$-equivariant root problem: $\varphi(g)=e K$.

Let $K_{1}, \ldots, K_{\left|\pi_{0}(K)\right|}$ denote the connected components of $K, \operatorname{dim} K=k$, $\operatorname{dim} M=n$ and so $\operatorname{dim} G=n+k$. Without loss of generality, we may assume that $f$ has a finite number of fixed points in $M$. For an isolated fixed point $g K$ of $f$, let $\mathcal{O}_{g}$ be the isolated $K$-orbit of roots of $\varphi$ containing $g$. Consider the diagram

$$
(G, G-K) \stackrel{\theta_{g}}{\rightarrow}\left(G, G-\mathcal{O}_{g}\right) \stackrel{i}{\longleftrightarrow}\left(V_{g}, V_{g}-\mathcal{O}_{g}\right) \stackrel{\varphi}{\rightarrow}(M, M-e K) \stackrel{j}{\longleftarrow} M
$$

where $V_{g}$ is a closed $K$-invariant neighborhood of $\mathcal{O}_{g}$ containing no other roots of $\varphi$, and $\theta_{g}$ is the homeomorphism induced by $g$. This defines an integer-valued equivariant root index $\omega_{K}\left(\varphi, \mathcal{O}_{g}\right)$. For each $i, 1 \leq i \leq\left|\pi_{0}(K)\right|$, we let $\mathcal{O}_{g}^{i}=\mathcal{O}_{g} \cap K_{i}$ and the equivariant root index of the $i$ th component $\mathcal{O}_{g}^{i}$ is simply

$$
\omega_{K}\left(\varphi, \mathcal{O}_{g}^{i}\right)=\frac{1}{\left|\pi_{0}(K)\right|} \omega_{K}\left(\varphi, \mathcal{O}_{g}\right)
$$

The connection between the equivariant root index of $\varphi$ and the fixed point index of $f$ is given by the following

Lemma 1.2 ([16, Lemma 2.3]). Given a selfmap $f: M \rightarrow M$ and its corresponding $K$-map $\varphi: G \rightarrow M$, if $g K$ is an isolated fixed point of $f$, then $\omega_{K}\left(\varphi, \mathcal{O}_{g}^{1}\right)=(-1)^{n} \operatorname{ind}(f, g K)$ where $n=\operatorname{dim} M$.

REMARK 1. We take this opportunity to correct Lemma 2.3 of [16]. The extra quantity $(-1)^{n}$ should be added. The computation there used the definition of the Lefschetz number incorrectly since $L(1, f)=(-1)^{n} L(f, 1)=$ $(-1)^{n} L(f)$. 
In Section 2, we show that any two $K$-orbits of roots of $\varphi$ are related by a homotopy and hence that their equivariant root index must be of the same sign, yielding Theorem 2.2.

The existence of a fixed point free map homotopic to $f: M \rightarrow M$ is equivalent to the vanishing of the obstruction $o(f)$ to deforming the graph of $1 \times f$ off the diagonal of $M$. In fact, this obstruction has a cochain representation in terms of the fixed point indices of the essential fixed point classes of $f$ (Theorem 4.3 of [4]). In Section 3, we consider the (primary) equivariant obstruction $o_{K}(\varphi)$ to deforming $\varphi$ equivariantly to a root free map, analogous to $o(f)$. We employ the Bredon-Illman cohomology with local coefficients developed in [11]. The appropriate equivariant local coefficient system admits an action from the equivariant fundamental groupoid whose typical object group is the fundamental group of a transformation group of F. Rhodes [14]. This action coincides with the action of the extension group $\Gamma_{G}$ on $\pi$ as described in [16]. We show that $o_{K}(\varphi)=0$ iff $o(f)=0$. Furthermore, $o_{K}(\varphi)$ has a cochain representation in terms of the equivariant root indices of the $K$-Nielsen root classes of $\varphi$.

The relationship between $o(f)$ and $N(f)$ gives rise to the connection between $o(f)$ and $\mathcal{L}(f, \widetilde{f})$, the Reidemeister trace of $f$ (with respect to the lift $\widetilde{f}$ ). The Reidemeister trace (also known as the generalized Lefschetz number in [4] and in [9]) is an alternating sum of traces involving the integral group ring $\mathbb{Z} \pi$ where $\pi$ is the group of deck transformations of the universal cover of $M$. The Reidemeister trace contains information on both the Lefschetz and the Nielsen numbers so that

$$
o(f)=0 \Leftrightarrow \mathcal{L}(f, \widetilde{f})=0 .
$$

In the last section, we define an equivariant (Reidemeister) degree $\mathcal{D}_{K}(\varphi, \widetilde{\varphi})$, analogous to the Reidemeister trace $\mathcal{L}(f, \widetilde{f})$. A simple formula relating $\mathcal{L}(f, \widetilde{f})$ and $\mathcal{D}_{K}(\varphi, \widetilde{\varphi})$ is given; it generalizes a similar formula obtained in [16] when $K$ is a finite subgroup. The connection between $\mathcal{D}_{K}(\varphi, \widetilde{\varphi})$ and the equivariant obstruction $o_{K}(\varphi)$, similar to that between $\mathcal{L}(f, \widetilde{f})$ and $o(f)$ as in [4], will also be given. By defining the notion of an equivariant Nielsen number for roots, we obtain a similar description of $\mathcal{D}_{K}(\varphi, \widetilde{\varphi})$ such that the sum of the coefficients yields the sum of the equivariant root indices, and the sum of the number of classes yields the equivariant Nielsen root number.

2. Computation of $\omega_{K}(\varphi, \mathcal{O})$. In this section, we compute the equivariant root index $\omega_{K}(\varphi, \mathcal{O})$ and show that the equivariant root indices are all zero or have the same sign. We prove our main result, Theorem 2.2, which states that orientable homogeneous spaces of compact connected Lie groups are Jiang type spaces. 
Let $\mathcal{O}_{1}, \ldots, \mathcal{O}_{m}$ be the root orbits of $\varphi$ and $g_{1} K, \ldots, g_{m} K$ be the corresponding fixed points of $f$.

LEMMA 2.1. If $\omega_{K}\left(\varphi, \mathcal{O}_{i}\right)=0$ for some $i$, then $\omega_{K}\left(\varphi, \mathcal{O}_{j}\right)=0$ for all $j$, $1 \leq j \leq\left|\pi_{0}(K)\right|$. If $\omega_{K}\left(\varphi, \mathcal{O}_{i}\right) \neq 0$, then

$$
\omega_{K}\left(\varphi, \mathcal{O}_{i}\right) \cdot \omega_{K}\left(\varphi, \mathcal{O}_{j}\right)>0 \quad \text { for all } j, 1 \leq j \leq\left|\pi_{0}(K)\right| \text {. }
$$

Proof. Without loss of generality, we may assume that each $g_{i} K$ is a distinct fixed point class of $f$ and thus the $\mathcal{O}_{i}$ 's are distinct $K$-orbit root classes of $\varphi$.

Each $\mathcal{O}_{i}$ is a disjoint union of ordinary root classes of $\varphi$. In fact, if $\gamma_{1}$ and $\gamma_{2}$ are root classes of $\varphi$ so that $\gamma_{1}$ and $\gamma_{2}$ belong to the same $\mathcal{O}$ then there exists a $\sigma \in K$ such that $\gamma_{2}=\sigma \gamma_{1}$. Conversely, if $\alpha$ is an ordinary root class and $\alpha \subset \mathcal{O}_{i}$, then $\alpha$ is a disjoint union of components of $\mathcal{O}_{i}$. Since $\omega_{K}\left(\varphi, \mathcal{O}_{i}^{i_{1}}\right)=\omega_{K}\left(\varphi, \mathcal{O}_{i}^{i_{2}}\right)$ for any two components $\mathcal{O}_{i}^{i_{1}}$ and $\mathcal{O}_{i}^{i_{2}}$ of $\mathcal{O}_{i}$, one can define $\omega_{K}(\varphi, \alpha)$ to be $k_{\alpha} \cdot \omega_{K}\left(\varphi, \mathcal{O}_{i}^{1}\right)$ where $k_{\alpha}$ denotes the number of connected components in $\alpha$.

Let $\alpha \subset \mathcal{O}_{i}, \beta \subset \mathcal{O}_{j}$ be two distinct root classes of $\varphi$. Let $x \in \alpha, y \in \beta$ and $C$ be a path in $G$ with $C(0)=y, C(1)=x$. Since $M$ is a manifold, there exists an isotopy $H: M \times[0,1] \rightarrow M$ such that $H_{1}=1_{M}$ and $H_{t}(e K)=$ $\varphi \circ C(t)$. Let $F: G \times[0,1] \rightarrow M$ be given by $F_{t}=H_{t} \circ \varphi$. Now,

$$
\begin{aligned}
\left\{F_{t}(C(1-t))\right\} & =\left\{H_{t} \circ \varphi \circ C(1-t)\right\} \\
& =\left\{H_{t} \circ H_{1-t}(e K)\right\} \sim\{e K\} \quad \text { (relative to endpoints). }
\end{aligned}
$$

This implies that $\alpha$ and $\beta$ are $F$-related and thus they belong to the same root class $\mathcal{N}$ of $F$.

Let $\mathcal{N}_{t}=p_{1}(\mathcal{N} \cap(G \times\{t\}))$ where $p_{1}(w, s)=w, s \in[0,1]$. Note that $\alpha=\mathcal{N}_{0}$ and $\beta=\mathcal{N}_{1}$. Choose an open neighborhood $W$ of $\mathcal{N}$ in $G \times[0,1]$ so that $\operatorname{cl}(W)$ does not contain any roots of $F$ other than $\mathcal{N}$. For any $r$, $0 \leq r \leq 1$, let

$$
W_{r}=p_{1}(W \cap(G \times\{r\})), \quad K_{r}=p_{1}\left(F^{-1}(e K)\right)-W_{r} .
$$

Since $K_{r}$ is compact, by uniform continuity, for any $\varepsilon>0$, there exists $\delta>0$ such that

$$
|r-s|<\delta \Rightarrow\left|F_{r}(z)-F_{s}(z)\right|<\varepsilon, \forall z \in K_{r} .
$$

Thus, $F_{s}$ has no roots in $K_{r}$ and so $\mathcal{N}_{s} \subset W_{r}$.

Define $\left\{\Gamma_{t}^{r, s}\right\}: G \rightarrow M$ by

$$
\Gamma_{t}^{r, s}(z)=F(z,(1-t) r+t s) .
$$

Then, $\Gamma_{0}^{r, s}=F_{r}, \Gamma_{1}^{r, s}=F_{s}$ and

is compact in $W_{r}$.

$$
\bigcup_{0 \leq t \leq 1}\left(\Gamma_{t}^{r, s}\right)^{-1}(e K) \cap W_{r}
$$


Next, we show that $\varphi_{*}\left(o_{\alpha}\right)=\varphi_{*}\left(o_{\beta}\right)$ where $o_{\alpha}$ and $o_{\beta}$ denote the fundamental homology classes around $\alpha$ and $\beta$ respectively. To see that, we observe that for any $t \in[0,1]$, the inclusion

$$
j:\left(W_{r}, W_{r}-\bigcup_{0 \leq \tau \leq 1}\left(\Gamma_{\tau}^{r, s}\right)^{-1}(e K)\right) \hookrightarrow\left(W_{r}, W_{r}-\left(\Gamma_{t}^{r, s}\right)^{-1}(e K)\right)
$$

induces on homology a homomorphism $j_{*}$ which takes $o_{1}$ to $o_{2}$ where

$$
\begin{aligned}
& o_{1}=\text { fundamental homology class around } \underset{0 \leq \tau \leq 1}{\bigcup}\left(\Gamma_{\tau}^{r, s}\right)^{-1}(e K) \cap W_{r}, \\
& o_{2}=\text { fundamental homology class around }\left(\Gamma_{t}^{r, s}\right)^{-1}(e K) \cap W_{r} .
\end{aligned}
$$

Since

$$
\left\{\left.\Gamma_{t}^{r, s}\right|_{W_{r}}\right\}:\left(W_{r}, W_{r}-\bigcup_{0 \leq \tau \leq 1}\left(\Gamma_{\tau}^{r, s}\right)^{-1}(e K)\right) \rightarrow(M, M-e K)
$$

is a homotopy, it follows that

$$
\left(\left.\Gamma_{0}^{r, s}\right|_{W_{r}}\right)_{*}\left(o_{1}\right)=\left(\left.\Gamma_{1}^{r, s}\right|_{W_{r}}\right)_{*}\left(o_{1}\right) .
$$

Together with excision, we obtain

$$
\left(F_{r}\right)_{*} \circ i_{*}^{-1}\left(o_{1}\right)=\left(F_{s}\right)_{*} \circ i_{*}^{-1}\left(o_{1}\right)
$$

where

$$
i:\left(W_{r}, W_{r}-\bigcup_{0 \leq \tau \leq 1}\left(\Gamma_{\tau}^{r, s}\right)^{-1}(e K)\right) \rightarrow\left(G, G-\mathcal{N}_{z}\right)
$$

is the inclusion. By the compactness of $[0,1]$, we conclude that $F_{0_{*}}=F_{1_{*}}$, which then implies that

$$
\left(H_{0} \circ \varphi\right)_{*}\left(o_{\alpha}\right)=\varphi_{*}\left(o_{\beta}\right)
$$

where $o_{\alpha}$ and $o_{\beta}$ denote the fundamental homology classes around $\alpha$ and $\beta$ respectively.

Since $H_{t}$ is an isotopy and $M$ is orientable, we have

$$
\left(H_{0} \circ \varphi\right)_{*}=\varphi_{*} \text {. }
$$

In other words,

$$
\omega_{K}(\varphi, \alpha)=\omega_{K}(\varphi, \beta)
$$

Since two roots in the same connected component of $\mathcal{O}_{i}$ are Nielsen equivalent as ordinary roots of $\varphi, \alpha$ consists of a disjoint union of connected components of $\mathcal{O}_{i}$, all homeomorphic to one other. We conclude that

$$
\omega_{K}\left(\varphi, \mathcal{O}_{i}\right)=0 \Leftrightarrow \omega_{K}(\varphi, \alpha)=0 \Leftrightarrow \varphi_{*}\left(o_{\alpha}\right)=0 \Leftrightarrow \omega_{K}\left(\varphi, \mathcal{O}_{j}\right)=0 .
$$

Moreover,

$$
\omega_{K}(\varphi, \alpha)=k_{\alpha} \cdot \omega_{K}\left(\varphi, \mathcal{O}_{i}^{1}\right), \quad \omega_{K}(\varphi, \beta)=k_{\beta} \cdot \omega_{K}\left(\varphi, \mathcal{O}_{j}^{1}\right)
$$


for some positive integers $k_{\alpha}, k_{\beta}$. Since

$$
\omega_{K}\left(\varphi, \mathcal{O}_{i}\right)=\left|\pi_{0}(K)\right| \cdot \omega_{K}\left(\varphi, \mathcal{O}_{i}^{1}\right),
$$

it follows that

$$
\omega_{K}\left(\varphi, \mathcal{O}_{i}\right)=\frac{\left|\pi_{0}(K)\right|}{k_{\alpha}} \cdot \omega_{K}(\varphi, \alpha), \quad \omega_{K}\left(\varphi, \mathcal{O}_{j}\right)=\frac{\left|\pi_{0}(K)\right|}{k_{\beta}} \cdot \omega_{K}(\varphi, \beta) .
$$

Hence, if $\omega_{K}\left(\varphi, \mathcal{O}_{i}\right) \neq 0$ then $\omega_{K}\left(\varphi, \mathcal{O}_{i}\right) \cdot \omega_{K}\left(\varphi, \mathcal{O}_{j}\right)>0$.

Now, we prove our main result.

THEOREM 2.2. Let $G$ be a compact connected Lie group, $K$ a closed subgroup and $M=G / K$ the homogeneous space of right cosets. Assume that $M$ is orientable. For any $f: M \rightarrow M$, either $(1) L(f)=0 \Rightarrow N(f)=0$; or (2) $L(f) \neq 0 \Rightarrow N(f)=R(f)$. Furthermore, $L(f)=0$ implies that $f$ is homotopic to a fixed point free map.

Proof. It follows from Lemma 1.2 that

$$
\omega_{K}\left(\varphi, \mathcal{O}_{i}\right)=(-1)^{n}\left|\pi_{0}(K)\right| \cdot \operatorname{ind}\left(f, g_{i} K\right)
$$

for $1 \leq i \leq\left|\pi_{0}(K)\right|$. By Lemma 2.1, we conclude that either ind $\left(f, g_{i} K\right)=0$ for all $i$ or the fixed point indices are all nonzero of the same sign. Thus, $L(f)=0 \Rightarrow N(f)=0$, and when $L(f) \neq 0$, the argument in the proof of Theorem 5.3 of [16] yields the equality $N(f)=R(f)$. The assertion that $f$ is deformable to be fixed point free when $L(f)=0$ follows from Theorem 4.2 and Proposition 3.4 of [16] with one additional case to consider. This additional case is when $M$ is the 2-sphere, which is clearly Wecken. Hence, the proof is complete.

REMARK 2. Theorem 2.2 is clearly false if $M$ is nonorientable, e.g., take $M$ to be an even-dimensional real projective space. This result does not hold for orientable homogeneous spaces of noncompact Lie groups in general. There exist orientable solvmanifolds for which neither (1) nor (2) holds (see, e.g., [8]).

3. The equivariant obstruction $o_{K}(\varphi)$. In this section, we compute the (primary) equivariant obstruction to deformation $o_{K}(\varphi)$ and show that it vanishes if and only if the primary obstruction to deforming $f$ to be fixed point free $o(f)$ vanishes. There are a number of different equivariant obstruction theories developed for different purposes. Since we are in the equivariant setting of the situation as in [4], equivariant local coefficients must be employed. Therefore, we employ the one developed in [11]. We should point out that an equivariant cohomology theory with equivariant local coefficients was developed in [10], which then turned out to coincide with that in [11] for discrete groups (see [12]). We follow the obstruction theory for deformation as presented in [4] and in [11]. 
First, we replace the inclusion $i: M-e K \hookrightarrow M$ by a fiber map $q: E \rightarrow$ $M$ given by $q(x, \omega)=\omega(1)$ where $E=\left\{(x, \omega) \in M \times M^{[0,1]} \mid \omega(0)=x\right\}$ with compact-open topology. If $y \in M$ then $q^{-1}(y)=F=\{(x, \omega) \mid \omega(1)=y\}$. Choose a base point $x_{0} \in M-e K$. It follows that

$$
\pi_{k}\left(F,\left(x, \bar{x}_{0}\right)\right) \cong \pi_{k+1}\left(M, M-e K, x_{0}\right) .
$$

If $n=\operatorname{dim} M \geq 3$ then $F$ is $(n-2)$-connected and hence $F$ is $q$-simple for all $q>0$. Note that $E$ is a $K$-space. Given a $K$-map $\varphi: G \rightarrow M, \varphi$ is $K$-deformable into $M-e K$ iff there is a solution $\psi$ such that the following diagram is commutative:

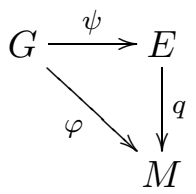

That is, there exists a $K$-map $\psi: G \rightarrow E$ such that $q \circ \psi=\varphi$. Following [11], there is an equivariant (primary) obstruction $o_{K}(\varphi)$ to finding such a $K$-map $\psi$.

One can often regard a root problem as a coincidence problem with one of the maps being a constant map. Thus, we obtain a $K$-fibration $p: \mathcal{E} \rightarrow$ $M \times M$ by replacing $M \times M-\Delta \hookrightarrow M \times M$. Given a $K$-map $\varphi: G \rightarrow M$, one can show, using an elementary argument about the equivariant covering homotopy property of a $K$-fibration, that $\varphi \times \overline{e K}: G \rightarrow M \times M$ is $K$ deformable into $M \times M-\Delta$ iff $\varphi$ is $K$-deformable into $M-e K$. Denote by $o_{K}(\varphi \times \overline{e K})$ the corresponding obstruction.

In order to define $o_{K}(\varphi)$ (or $o_{K}(\varphi \times \overline{e K})$ ), we first recall the BredonIllman cohomology with local coefficients as developed in [11].

Let $X$ be a $K$-space. Define a category $\Pi_{K}(X)$ as follows. The objects of $\Pi_{K}(X)$ are $K$-maps $x_{H}: K / H \rightarrow X$ and a morphism from $x_{H}: K / H \rightarrow X$ to $y_{L}: K / L \rightarrow X$ is a pair $(\widehat{k},[\phi])$ where $\widehat{k}: K / H \rightarrow K / L$ is the $K$-map corresponding to $k^{-1} H k \subseteq L$ and $[\phi]$ is the $K$-homotopy class of the $K$ homotopy $\phi: K / H \times[0,1] \rightarrow X$ from $x_{H}$ to $y_{L} \circ \widehat{k}$. Then an equivariant local (coefficient) system is simply a contravariant functor $\Gamma: \Pi_{K}(X) \rightarrow \mathcal{A} b$ where $\mathcal{A} b$ denotes the category of abelian groups.

Since there is a natural bijection between $\operatorname{Map}_{K}(K / H, X)$ and $X^{H}$, an object $x_{H}$ in $\Pi_{K}(X)$ may be identified with a point in $X^{H}$ and so if $(\widehat{k},[\phi])$ is a morphism then $\phi$ may be identified with a path in $X^{H}$ from $x_{H}$ to $y_{L} \circ \widehat{k}$ where $\widehat{k}: K / H \rightarrow K / L$. Since $K$-maps from $K / L$ to $K / H$ need not exist, $\Pi_{K}(X)$ is not a groupoid in general.

Given an equivariant local system $\Gamma$ on a $K$-space $X$, let $S_{K}^{n}(X ; \Gamma)$ be the group of all functions $c$ which map an equivariant $n$-simplex $\sigma$ : $\Delta_{n} \times K / H \rightarrow X$ to an element of $\Gamma(\bar{\sigma})$ where $\bar{\sigma}$ is the restriction of $\sigma$ to the 
first vertex of $\Delta_{n}$. Following [11] (see also [12] and [10]), the cohomology

$$
\mathfrak{H}_{K}^{*}(X ; \Gamma):=H^{*}\left(S_{K}^{*}(X ; \Gamma)\right)
$$

is called the Bredon-Illman cohomology of $X$ with local coefficients $\Gamma$.

In the nonequivariant situation, the obstruction to deforming a map to be fixed point (or coincidence) free was calculated in [4] as follows. First consider the path fibration $p: \mathcal{E} \rightarrow M \times M$ induced by replacing the inclusion map $M \times M-\Delta \hookrightarrow M \times M$. The fiber $\mathcal{F}$ of $p$ gives rise to a local system $\pi_{n-1}(\mathcal{F}) \cong \mathbb{Z} \pi$ and the action of $\pi_{1}(M \times M) \equiv \pi \times \pi$ on $\pi_{n-1}(\mathcal{F})$ is given by the action of $\pi \times \pi$ on $\pi$ via

$$
(\sigma, \tau) \bullet \alpha \mapsto \tau \alpha \sigma^{-1} .
$$

For the coincidence situation (see e.g. [7]), the primary obstruction $o_{n}(f, g)$ to deforming $f, g: Z \rightarrow M$ to be coincidence free on the $n$th skeleton of $Z$, $n=\operatorname{dim} M$, lies in $H^{n}\left(Z ; \mathbb{Z} \pi^{*}\right)$ where $\mathbb{Z} \pi^{*}$ is the local system induced by pulling back $\mathbb{Z} \pi$ over $M \times M$ by $f \times g$. Furthermore, the action of $\pi_{1}(Z)$ on $\mathbb{Z} \pi^{*}$ is given by

$$
\sigma \bullet \alpha \mapsto \psi_{2}(\sigma) \alpha \psi_{1}(\sigma)^{-1}
$$

where $\sigma \in \pi_{1}(Z)$, and $\psi_{1}, \psi_{2}: \pi_{1}(Z) \rightarrow \pi$ are the induced homomorphisms of $f$ and of $g$ respectively.

Consider the $K$-map $\varphi \times \overline{e K}: G \rightarrow M \times M$ where $\overline{e K}$ denotes the constant map at $e K \in M$. Then the pullback $(\varphi \times \overline{e K})^{*}\left(\pi_{n}(\mathcal{F})\right)$ is an equivariant local system on $G$. Since $G$ is a free $K$-space, the category $\Pi_{K}(G)$ is in fact a groupoid. Furthermore, $\Pi_{K}(G)$ is the semidirect product groupoid or the fundamental groupoid of the transformation group $(G, K)$ of [2]. It was pointed out in [2] that a typical object group is the fundamental group of the transformation group, denoted by $\sigma(G, e, K)$, introduced by $\mathrm{F}$. Rhodes in [14] (for generalization of Rhodes groups and their connection with equivariant fixed point theory, see [6]). Thus, in order to compute the obstruction to $K$-deformation, we need to know how $\sigma(G, e, K)$ acts on $\pi$ and hence on $\mathbb{Z} \pi \cong(\varphi \times \overline{e K})^{*}\left(\pi_{n-1}(\mathcal{F})\right)$. In other words, the action (3.2) must be modified by replacing $\psi_{1}$ and $\psi_{2}$ with the appropriate homomorphisms.

Given a $K$-space $X$ and a base point $x_{0}$, the fundamental group of the transformation group $(X, K)$ is, by definition, the group of homotopy classes of the form $[k ; \alpha]$ where $\alpha$ is a path from $x_{0}$ to $k x_{0}$, with multiplication given by

$$
\left[k_{1} ; \alpha_{1}\right] *\left[k_{2} ; \alpha_{2}\right]:=\left[k_{1} k_{2} ; \alpha_{1}+k_{1} \alpha_{2}\right] .
$$

By considering the universal cover $\widetilde{X}$ as the space of all path classes $[\alpha]$ of paths $\alpha$ beginning at $x_{0}$, and the covering map $\eta: \widetilde{X} \rightarrow X$ which sends a point $[\alpha]$ to $\alpha(1)$, the endpoint of $\alpha$, it is straightforward to show that there is a one-to-one correspondence between $[k ; \alpha]$ and the unique lift $\widetilde{k}$ of $k$ such 
that $\widetilde{k}\left(\left[\bar{x}_{0}\right]\right)=[\alpha]$ where $\bar{x}_{0}$ is the constant path at $x_{0}$. This bijection is a group isomorphism between $\sigma\left(X, x_{0}, K\right)$ and the extension group $\widetilde{K}$ given by

$$
\widetilde{K}=\{\widetilde{k} \in \operatorname{Homeo}(\widetilde{X}) \mid \eta \widetilde{k}=k \eta \text { for some } k \in K\} .
$$

In [16], $\widetilde{K}=\Gamma_{M}$ when $X=M$ and $\widetilde{K}=\Gamma_{G}$ when $X=G$.

Now in our situation, the $K$-map $\varphi: G \rightarrow M$ induces a homomorphism $\Psi: \Gamma_{G} \rightarrow \Gamma_{M}$ (but not to $\pi$ ). Similarly, the constant map $\overline{e K}: G \rightarrow M$ also induces a map $\widetilde{\tau}: \Gamma_{G} \rightarrow \Gamma_{M}$ so that for any $\sigma \in \Gamma_{G}, \widetilde{\tau}(\sigma)=[\bar{\sigma} ; \overline{e K}]$ where $\bar{\sigma} \in K$ is covered by $\sigma$. Hence, the action (3.2) now becomes

$$
\sigma \bullet \alpha \mapsto \widetilde{\tau}(\sigma) \alpha \Psi(\sigma)^{-1} .
$$

This action coincides with the action given on p. 36 of [16] (the $\tau(\bar{\sigma})$ there is the same element as the $\widetilde{\tau}(\sigma)$ given here).

The $K$-action on $G$ gives rise to a $K$-equivariant $\mathrm{CW}$ decomposition on $G$ by attaching equivariant cells of type $K$ (since the $K$-action is free). More precisely, there is a filtration

$$
G(0) \subset G(1) \subset \cdots \subset G(n)=G
$$

where $G(0)=\emptyset$ and $G(i)$ is obtained from $G(i-1)$ by attaching free $i$ cells. Note that the $K$-equivariant cellular dimension of $G$ is $n$, which is the dimension of $M=G / K$. For each $i$, a typical $i$-cell is given by the $K$-(characteristic) map

$$
\left(e^{i} \times K, \partial e^{i} \times K\right) \rightarrow(G(i), G(i-1))
$$

where $e^{i}$ is a typical (nonequivariant) $i$-cell in $M=G / K$ and is homeomorphic to the unit $i$-ball in $\mathbb{R}^{i}$. The $K$-action on $\left(e^{i}, \partial e^{i}\right)$ is trivial. One can define $K$-equivariant cellular cochains and show that the corresponding cohomology group is isomorphic to $\mathfrak{H}_{K}^{*}(X ; \Gamma)$ (see Theorem 7.3 of [11]).

Suppose $n=\operatorname{dim} M \geq 3$ (if $\operatorname{dim} M=2$, then $M$ must be either the 2sphere or the torus). Every selfmap $f: M \rightarrow M$ is homotopic to a map with a finite number of fixed points each of which lies in the interior of an $n$-cell. Equivalently, the corresponding $K$-map is $K$-homotopic to a $K$-map with a finite number of root orbits each of which lies inside an equivariant $n$-cell. Thus, without loss of generality, we may assume that $\varphi$ already has this property, i.e., $\varphi$ is root free on $G(n-1)$. The map $\varphi \times \overline{e K}: G \rightarrow M \times M$ has an equivariant partial lifting $\varphi_{n-1} \times \overline{e K}: G(n-1) \rightarrow \mathcal{E}$ such that $p \circ\left(\varphi_{n-1} \times \overline{e K}\right)=\left.(\varphi \times \overline{e K})\right|_{G(n-1)}$. Therefore, following [15], there is a primary equivariant obstruction to extending $\varphi_{n-1}$ to all of $G$. Thus the equivariant obstruction we consider is the one for $\varphi \times \overline{e K}$ and is given by

$$
o_{K}^{n}(\varphi \times \overline{e K}) \in \mathfrak{H}_{K}^{n}\left(G ;(\varphi \times \overline{e K})^{*} \pi_{n-1}(\mathcal{F})\right) .
$$

Next, we compute this obstruction by giving a cochain representation $c_{K}^{n}(\varphi \times \overline{e K})$ in terms of the equivariant root indices of $\varphi$. 
Given an equivariant $n$-cell $\sigma: \Delta_{n} \times K \rightarrow G$, we consider its corresponding nonequivariant $n$-cell $\widetilde{\sigma}: \Delta_{n} \rightarrow G$ given by $\widetilde{\sigma}(x)=\sigma(x, e)$. Then $\left.(\varphi \times \overline{e K}) \circ \widetilde{\sigma}\right|_{\partial \Delta_{n}}$ gives rise to a function

$$
c_{K}^{n}(\varphi \times \overline{e K})(\sigma)=\left[\left.(\varphi \times \overline{e K}) \circ \widetilde{\sigma}\right|_{\partial \Delta_{n}}\right]
$$

defined on equivariant $n$-cells $\sigma$ with values in $(\varphi \times \overline{e K})^{*} \pi_{n-1}(\mathcal{F})$. In fact, $c_{K}^{n}(\varphi \times \overline{e K})$ is a cocycle representing the primary obstruction $o_{K}^{n}(\varphi \times \overline{e K})$. Following pp. $65-70$ of [4] and using the identification $\pi_{n-1}(\mathcal{F}) \cong$ $\pi_{n-1}(M, M-e K)$, we have

$$
c_{K}^{n}(\varphi \times \overline{e K})(\sigma)= \begin{cases}0 & \text { if } \varphi \text { is root free on } \sigma, \\ \omega_{K}\left(\varphi, \mathcal{O}_{g}\right)\left[\varphi_{\sigma}^{\prime}\right] & \text { if } \mathcal{O}_{g} \subset \sigma .\end{cases}
$$

Here $\varphi_{\sigma}^{\prime}$ represents the composite $\left.\varphi\right|_{V_{g}} \circ i_{\sigma}$ where

$$
i_{\sigma}:(\sigma, \partial \sigma) \rightarrow\left(V_{g}, V_{g}-\mathcal{O}_{g}\right)
$$

sends a fixed orientation to the orientation on $V_{g}$. Hence, we have

Theorem 3.1. The cocycle $c_{K}^{n}(\varphi \times \overline{e K})$ is given by

$$
c_{K}^{n}(\varphi \times \overline{e K})=\sum_{\sigma} \omega_{K}\left(\varphi, \mathcal{O}_{g}\right)\left[\varphi_{\sigma}^{\prime}\right] .
$$

4. Reidemeister trace and equivariant Reidemeister degree. In [4], the Nielsen number $N(f)$ and the obstruction $o(f)$ were related to the Reidemeister trace $\mathcal{L}(f, \widetilde{f})$ (also called the generalized Lefschetz number in [4]). In this final section, we define a similar element $\mathcal{D}_{K}(\varphi, \widetilde{\varphi})$, which corresponds to $\mathcal{L}(f, \widetilde{f})$, in the free $\mathbb{Z}$-module generated by the equivariant Reidemeister root orbit classes of $\varphi$.

Let $\eta_{M}: \widetilde{M} \rightarrow M$ be the universal cover of $M$. Denote by $\pi$ the group of covering transformations which can be identified with $\pi_{1}(M)$. Suppose that $\widetilde{f}: \widetilde{M} \rightarrow \widetilde{M}$ is a lift of $f$. Given any $\sigma \in \pi$ there exists a unique element $\phi(\sigma) \in \pi$ such that $\tilde{f} \circ \sigma=\phi(\sigma) \circ \widetilde{f}$. This defines a group homomorphism $\phi: \pi \rightarrow \pi$ and hence an action of $\pi$ on $\pi$ by

$$
\sigma \bullet \alpha=\sigma \alpha \phi(\sigma)^{-1} \quad \text { for } \alpha, \sigma \in \pi .
$$

Let $\mathbb{Z} \mathcal{R}_{\phi}[\pi]$ be the free $\mathbb{Z}$-module generated by the set $\mathcal{R}_{\phi}[\pi]$ of orbits of the Reidemeister action (4.1). The Reidemeister trace $\mathcal{L}(f, \widetilde{f})$ is defined to be an alternating sum of traces at the chain level in the universal cover and it can be written (see $[4$, p. 89]) as a weighted sum so that

$$
\mathcal{L}(f, \widetilde{f})=\sum_{\varrho \in \mathcal{R}_{\phi}[\pi]} \operatorname{ind}\left(f, \mathbf{F}_{\varrho}\right) \varrho \in \mathbb{Z} \mathcal{R}_{\phi}[\pi]
$$

where $\mathbf{F}_{\varrho}$ is the fixed point class associated to the Reidemeister class $\varrho$. Note that $\mathcal{L}(f, \widetilde{f})$ contains information on both the Nielsen and the Lefschetz 
numbers, and it enjoys the usual properties such as homotopy invariance, commutativity and homotopy type invariance (see $\S 6$ of [4] or [9]).

Denote by $\eta_{G}: \widetilde{G} \rightarrow G$ the universal cover. Let $\varphi: G \rightarrow M$ be a $K$-map and $f: M \rightarrow M$ its associated selfmap. Consider the following extension groups covering the $K$-actions. Let

$$
\begin{aligned}
\Gamma_{G} & =\left\{\widetilde{\gamma} \in \operatorname{Homeo}(\widetilde{G}) \mid \eta_{G} \widetilde{\gamma}=\gamma \eta_{G} \text { for some } \gamma \in K\right\}, \\
\Gamma_{M} & =\left\{\widetilde{\gamma} \in \operatorname{Homeo}(\widetilde{M}) \mid \eta_{M} \widetilde{\gamma}=\gamma \eta_{M} \text { for some } \gamma \in K\right\}
\end{aligned}
$$

Thus, we have the following exact sequences of groups:

$$
1 \rightarrow \pi_{1}(G) \equiv \operatorname{Cov}\left(\eta_{G}\right) \rightarrow \Gamma_{G} \rightarrow K \rightarrow 1
$$

and

$$
1 \rightarrow \pi_{1}(M) \equiv \operatorname{Cov}\left(\eta_{M}\right) \rightarrow \Gamma_{M} \rightarrow K \rightarrow 1
$$

For simplicity, we suppose $\varphi(e)=e K$ so that $f(e K)=e K$. Choose base points $\widetilde{e} \in \eta_{G}^{-1}(e), \widetilde{e K} \in \eta_{M}^{-1}(e K)$ and lifts

$$
\widetilde{\varphi}: \widetilde{G} \rightarrow \widetilde{M} \text { and } \tilde{f}: \widetilde{M} \rightarrow \widetilde{M}
$$

of $\varphi$ and $f$, respectively so that $\widetilde{\varphi}(\widetilde{e})=\widetilde{e K}$ and $\widetilde{f}(\widetilde{e K})=\widetilde{e K}$. For any $\sigma \in \Gamma_{G}$, there exists a unique element $\Psi(\sigma) \in \Gamma_{M}$ such that $\widetilde{\varphi} \sigma=\Psi(\sigma) \widetilde{\varphi}$. In fact, $\Psi: \Gamma_{G} \rightarrow \Gamma_{M}$ is a group homomorphism. Since $\gamma * e K=e K$ for all $\gamma \in K$, there exists a unique element $\tau(\gamma) \in \Gamma_{M}$ such that $\tau(\gamma)(\widetilde{e K})=\widetilde{e K}$ and $\tau(\gamma)$ covers $\gamma$. In particular, if $\gamma=e$, then $\tau(\gamma)=1_{\widetilde{M}}$. Thus, $\Gamma_{G}$ acts on $\pi_{1}(M)$ via

$$
\sigma \cdot \beta=\tau(\bar{\sigma}) \beta \Psi(\sigma)^{-1}
$$

where $\sigma \in \Gamma_{G}, \bar{\sigma} \in K$ is covered by $\sigma$, and $\beta \in \pi_{1}(M)$. We should point out that $\tau(\bar{\sigma})=\widetilde{\tau}(\sigma)$ so that the action (4.2) coincides with (3.3).

Denote by $\langle\beta\rangle_{K}$ the orbit of $\beta$ under the action given by (4.2). We call $\langle\beta\rangle_{K}$ the $K$-Reidemeister root orbit class of $\varphi$ containing $\beta$ and let $\Lambda_{\varphi}^{K}$ be the set of such classes. Lemma 5.2 of [16] gives a bijection between $\mathcal{R}_{\phi}[\pi]$ and $\Lambda_{\varphi}^{K}$. This bijection extends linearly to an isomorphism

$$
\Xi_{*}: \mathbb{Z} \mathcal{R}_{\phi}[\pi] \rightarrow \mathbb{Z} \Lambda_{\varphi}^{K}
$$

where $\mathbb{Z} \Lambda_{\varphi}^{K}$ denotes the free $\mathbb{Z}$-module generated by the set $\Lambda_{\varphi}^{K}$. On the one hand, $\mathbb{Z} \mathcal{R}_{\phi}[\pi]$ is obtained by the Reidemeister action (4.1), and on the other, $\Lambda_{\varphi}^{K}$ is obtained by the action of (4.2). In fact, we have the following commutative diagram of $\mathbb{Z}$-modules:

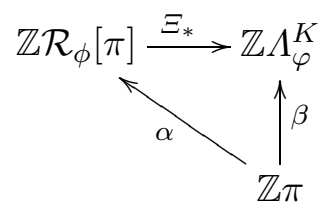


The map $\alpha$ is induced by the orbit map of the Reidemeister action (4.1) of $\pi$ on $\pi$ while the map $\beta$ is induced by the orbit map of the action (4.2) of $\Gamma_{G}$ on $\pi$.

For any $K$-map $\varphi: G \rightarrow M$, we define the $K$-Nielsen equivalence on $\varphi^{-1}(e K)$ as follows. Two roots $g_{1}, g_{2} \in \varphi^{-1}(e K)$ are said to be $K$-Nielsen equivalent as roots of $\varphi$ if either (i) $g_{2}=k * g_{1}=g_{1} k^{-1}$ for some $k \in K$ or (ii) there is a path $\sigma:[0,1] \rightarrow G$ with $\sigma(0)=g_{1}, \sigma(1)=\gamma * g_{2}$ for some $\gamma \in K$ such that $\varphi \circ \sigma$ is homotopic to the constant map $\overline{e K}$ relative to the endpoints. We call the equivalence classes $K$-Nielsen root classes; each of them consists of $K$-orbits of roots. It is easy to see that every such $K$-Nielsen root class is of the form $K \eta_{G}(\beta \widetilde{\varphi})^{-1}(\widetilde{e K})$ for some $\beta \in \pi_{1}(M)$. Given a $K$-Nielsen root class $\alpha$, we say that $\alpha$ is essential if the equivariant root index $\omega_{K}(\varphi, \alpha)$ is nonzero. Define the $K$-Nielsen root number to be

$$
N_{K}(\varphi ; e K):=\#\{\text { essential } K \text {-Nielsen root classes }\} \text {. }
$$

It is straightforward to verify that $N_{K}(\varphi ; e K)$ possesses the usual properties of the classical Nielsen number. In particular, it is invariant under $K$-equivariant homotopy. Define the $K$-Reidemeister degree of $\varphi$ to be

$$
\mathcal{D}_{K}(\varphi, \widetilde{\varphi})=\sum_{\langle\beta\rangle_{K} \in \Lambda_{\varphi}^{K}} \omega_{K}\left(\varphi, K \eta_{G}(\beta \widetilde{\varphi})^{-1}(\widetilde{e K})\right)\langle\beta\rangle_{K}
$$

where $K \eta_{G}(\beta \widetilde{\varphi})^{-1}(\widetilde{e K}) \subset \varphi^{-1}(e K)$ is the $K$-Nielsen root class of $\varphi$ corresponding to $\langle\beta\rangle_{K}$. By Lemma 1.2, we obtain the following

THEOREM 4.1.

$$
\Xi_{*}(\mathcal{L}(f, \tilde{f}))=(-1)^{n} \frac{\mathcal{D}_{K}(\varphi, \widetilde{\varphi})}{\left|\pi_{0}(K)\right|} .
$$

Define the $K$-equivariant degree of $\varphi$ to be

$$
\operatorname{deg}_{K} \varphi:=\sum \omega_{K}\left(\varphi, \mathcal{O}_{g}\right)
$$

the sum of the equivariant root indices of the Reidemeister root orbit classes. By Theorem 2.2, if one of the indices is nonzero, all the classes are essential, in which case the Reidemeister root orbit classes coincide with the essential Nielsen root classes.

The augmentation map $\mathbb{Z} \mathcal{R}_{\phi}[\pi] \rightarrow \mathbb{Z}$ sends $\mathcal{L}(f, \widetilde{f})$ to $L(f)$. Likewise, the augmentation map $\mathbb{Z} \Lambda_{\varphi}^{K} \rightarrow \mathbb{Z}$ sends $\mathcal{D}_{K}(\varphi, \widetilde{\varphi})$ to $\operatorname{deg}_{K} \varphi$. Thus, as a corollary of Theorem 4.1, we have

Corollary 4.2.

$$
L(f)=(-1)^{n} \frac{\operatorname{deg}_{K} \varphi}{\left|\pi_{0}(K)\right|} \quad \text { and } \quad N(f)=N_{K}(\varphi ; e K) .
$$


REMARK 3 . When $K$ is finite, $\omega_{K}\left(\varphi, \mathcal{O}_{g}\right)$ coincides with the ordinary root index $\omega\left(\varphi, \mathcal{O}_{g}\right)$ so that $\operatorname{deg}_{K} \varphi=\operatorname{deg} \varphi$. Thus Corollary 4.2 generalizes Corollary 4.5 of [16].

Given a map $f: X \rightarrow Y$ and a point $a \in Y$, the Nielsen root number of [1] was shown to be equal to either 0 or $\left[\pi_{1}(Y): f_{\#}\left(\pi_{1}(X)\right)\right]=R(f ; a)$ if $Y$ is a manifold. In our equivariant setting, we obtain the following similar result.

Theorem 4.3. Let $R_{K}(\varphi ; e K)$ denote the cardinality of $\Lambda_{\varphi}^{K}$. Then

$$
N_{K}(\varphi ; e K)= \begin{cases}R_{K}(\varphi ; e K) & \text { if } \omega_{K}\left(\varphi, \mathcal{O}_{g}\right) \neq 0 \text { for some root orbit } \mathcal{O}_{g}, \\ 0 & \text { otherwise. }\end{cases}
$$

Remark 4. To justify the use of the term degree in defining $\mathcal{D}_{K}(\varphi, \widetilde{\varphi})$, observe that the sum of the integral coefficients of $\mathcal{D}_{K}(\varphi, \widetilde{\varphi})$ is equal to

$$
\operatorname{deg}_{K} \varphi:=\sum \omega_{K}\left(\varphi, \mathcal{O}_{g}\right)
$$

where the sum ranges over the set of essential $K$-Nielsen root classes. As in the nonequivariant case, this equivariant degree $\operatorname{deg}_{K} \varphi$ gives an algebraic count of the number of $K$-orbits in the preimage $\varphi^{-1}(e K)$. Hence, the $K$-Reidemeister degree $\mathcal{D}_{K}(\varphi, \widetilde{\varphi})$ contains both the information on the $K$ Nielsen root number $N_{K}(\varphi ; e K)$ as well as the equivariant degree $\operatorname{deg}_{K} \varphi$. This description of $\mathcal{D}_{K}(\varphi, \widetilde{\varphi})$ in terms of $N_{K}(\varphi ; e K)$ and $\operatorname{deg}_{K} \varphi$ is completely analogous to that of the Reidemeister trace $\mathcal{L}(f, \widetilde{f})$ in terms of $N(f)$ and $L(f)$.

In [4], the obstruction $o(f)$ was explicitly calculated so that the fixed point index of each essential Nielsen class appears as the coefficient of the corresponding Reidemeister class in the Reidemeister trace. Similarly, from our calculation of $c_{K}^{n}(\varphi \times \overline{e K})$ in Theorem 3.1, we have the following

THEOREM 4.4 .

$$
o_{K}(\varphi)=0 \Leftrightarrow \mathcal{D}_{K}(\varphi, \widetilde{\varphi})=0 .
$$

REMARK 5. Although one can obtain Theorem 4.4 indirectly, using the fact that $o(f)=0$ iff $o_{K}(\varphi)=0$ and Theorem 4.1, the explicit representation of the equivariant obstruction cochain $c_{K}^{n}(\varphi \times \overline{e K})$ and Lemma 2.1 give a more direct proof.

\section{References}

[1] R. Brooks, Certain subgroups of the fundamental group and the number of roots of $f(x)=a$, Amer. J. Math. 95 (1973), 720-728. 
[2] R. Brown and P. Higgins, The fundamental groupoid of the quotient of a Hausdorff space by a discontinuous action of a discrete group is the orbit groupoid of the induced action, arXiv:math.AT/0212271, 2003.

[3] E. Fadell, Two vignettes in fixed point theory, in: Topological Fixed Point Theory and Applications (Tianjin, 1988), B. Jiang (ed.), Lecture Notes in Math. 1411, Springer, 1989, 46-51.

[4] E. Fadell and S. Husseini, Fixed point theory for non-simply connected manifolds, Topology 20 (1981), 53-92.

[5] D. Ferrario, On the equivariant Hopf theorem, Topology 42 (2003), 447-465.

[6] M. Golasiński, D. Gonçalves, and P. Wong, Equivariant evaluation subgroups and Rhodes groups, in preparation, 2005.

[7] D. Gonçalves, J. Jezierski and P. Wong, Obstruction theory and coincidences in positive codimension, Acta Math. Sinica, to appear.

[8] D. Gonçalves and P. Wong, Homogeneous spaces in coincidence theory, II, Forum Math. 17 (2005), 297-313.

[9] S. Husseini, Generalized Lefschetz numbers, Trans. Amer. Math. Soc. 272 (1982), $247-274$.

[10] I. Moerdijk and J.-A. Svensson, The equivariant Serre spectral sequence, Proc. Amer. Math. Soc. 118 (1993), 263-278.

[11] A. Mukherjee and G. Mukherjee, Bredon-Illman cohomology with local coefficients, Quart. J. Math. Oxford (2) 47 (1996), 199-219.

[12] G. Mukherjee and N. Pandey, Equivariant cohomology with local coefficients, Proc. Amer. Math. Soc. 130 (2001), 227-232.

[13] W. Lück, The equivariant degree, in: Algebraic Topology and Transformation Groups (Göttingen, 1987), Lecture Notes in Math. 1361, Springer, 1988, 123-166.

[14] F. Rhodes, On the fundamental group of a transformation group, Proc. London Math. Soc. 16 (1966), 635-650.

[15] T. tom Dieck, Transformation Groups, de Gruyter Stud. Math. 8, de Gruyter, Berlin, 1987.

[16] P. Wong, Fixed-point theory for homogeneous spaces, Amer. J. Math. 120 (1998), $23-42$.

Department of Mathematics

Bates College

Lewiston, ME 04240, U.S.A.

E-mail: pwong@bates.edu

Received 4 April 2005;

in revised form 5 July 2005 